\title{
Ecological research needed to manage risk and meet rising standards in mining rehabilitation
}

B Miller Botanic Gardens and Parks Authority, Kings Park, Australia

\begin{abstract}
Ecological sciences can assist mine planning and management on the way to effective rehabilitation and closure in a number of distinct ways. The identification of likely environmental impacts is a routine application that aids planning. Extending this to assessing the likely complexity of rehabilitation needs may be an equally valuable aid for risk-identification and mitigation, but is not at all routine. The identification of reference target ecosystems, the design of approaches to sample, assess and define these, and then to monitor rehabilitation trajectory towards them are elements of best practice. While these can significantly improve rehabilitation planning and effectiveness, they are often not applied in a way that enables this potential. Lastly, improving technical capacity to deliver required rehabilitation outcomes is the broadest area of potential benefit to closure management. Investing in environmental science may seem counterintuitive for a mining company but there are many potential benefits.
\end{abstract}

\section{Introduction}

Kings Park's Science Directorate has been involved in rehabilitation research in Western Australia (WA) and around the world for over three decades. We are currently engaged with research partnerships with more than 15 mining companies across WA. These projects employ 20 research scientists and support a similar number of graduate research students. In this keynote, I provide examples from this work to illustrate the outcomes and benefits of research in each of the above areas.

Pre-approval and industry best practice, often supported by regulatory requirements, involves biological survey to identify likely impacts of planned developments on biodiversity and other environmental values. Where significant values are identified such as threatened species or ecosystems, approvals managers may invest in research to clarify the distribution, identity, status and irreplaceability of these values. Research into genetic diversity, habitat or environmental associations and conservation threats may assist in determining acceptable levels of impact and/or potentially mitigating activities (Williams et al. 2014). This information supports or informs decisions around approval for development, but it also enables prediction of likely rehabilitation or closure conditions that may be imposed with mining approval, and how difficult or costly these may be to achieve.

Approval conditions often specify standards for the composition, structure, function, sustainability and resilience of rehabilitated communities at closure, especially where mining occurs in areas with significant conservation values (threatened species or ecological communities, valued waterways or conservation reserves), (see ed. Tibbett 2015). Different ecosystems involve different rehabilitation challenges resulting from the number of species they contain, the ease with which these species can be propagated or returned, and their requirements for specific rehabilitation environments or climate or disturbance events. Compounding this are the attributes of the site such as potential for topsoil return, time lags between disturbance and rehabilitation, altered hydrology or topography, the nature and extent of rehabilitation sites (tailings stores or waste dumps versus drill pads), the capacity of mine planning to optimise the availability and use of biological resources. 


\section{$2 \quad$ Assessing rehabilitation}

Rehabilitation goals can be specified as a condition of mining approval, derived from generic regulatory guidelines or driven by social expectations and corporate strategy. They vary from trivial to challenging.

Table 1 Key factors influencing the capacity of rehabilitation programmes to reach their goals

\begin{tabular}{l}
\hline External to control \\
\hline Imposed by regulation or corporate strategy \\
Attributes of the goals, i.e.: \\
- Stable cover. \\
- Stable cover using native species: \\
- Vegetation cover goals, e.g. $60 \%$ versus \\
- $90 \%$ cover. \\
- Thy representative local community. \\
- Species richness goals, e.g. $50 \%$ versus \\
$70 \%$ cover.
\end{tabular}

Within capacity to influence

Resulting from mine plans and activities at the site

The area concerned:

- Spatial extent.

- Diversity of communities or domains.

Resources available for rehabilitation

- Root zone subsoil.

- Topsoil:

- As a growing medium (volume, suitability).

- As a source of seed (collection, viability, storage and respreading conditions).

\section{Resulting from the attributes of the site or environment}

Attributes of the site, pre-mining:

- Richness of the community - how many species.

- The mix of species:

- Number that can be returned from topsoil seed.

- Number to be returned from seed.

- Number to be returned from greenstock:

- From seed.

- From cuttings.

- From tissue culture.

- Number with known germination techniques:

- Complexity and reliability of techniques.

- Number with known propagation techniques:

- Complexity and reliability of techniques.

Attributes of the site, post-mining:

- Appropriateness for the target community:

- Landform - exposure to radiation, wind, erosion, slope stability.

- site hydrology:

- Landform, soil texture and profiles to enhance infiltration and water retention.

- Substrate physical and chemical properties.

- Onsite threats (weeds, grazing, etc.).

Events:

- Reliance on episodic rainfall.

- Fire, severe drought and storms-erosion.

- Change in management or policy, downsizing.

- Adequate viable collected seed.

- Material (seed or cuttings) for propagation.

- Mulch, wood piles.

Ability to mobilise/manage resources:

- Scheduling in relation to season:

- Topsoil collection.

- Collection and storage of seed.

- Landforming and soil profile reconstruction.

- Site treatments (ripping, fertiliser, irrigation, mulch, etc.).

- Topsoil respreading.

- Propagation of greenstock.

- Seed treatments.

- Application of seed.

- Planting greenstock.

- Equipment and capacity:

- Landforming, ripping, irrigation.

- Propagation, nursery, seed store.

- Seed treatments.

- Seeding (direct seeding, broadcast seeding).

Attributes of the site, post-mining:

- Capacity to modify or amend post-mining conditions to suit the target community.

- Capacity to modify the target community to suit the post-mining conditions (or to compromise). 
The complexity of any given rehabilitation programme and its capacity to achieve its goals can depend on many factors (Table 1): some are manageable but many are not. Many of these unmanageable factors are known or could be known at different stages in mine development prior to clearing vegetation or commencement of activities. For instance, in WA planned significant impacts on existing very high conservation values (e.g. declared rare flora, threatened ecological communities, conservation reserves) are likely to lead to imposition of more demanding approval conditions. More rigorous conditions or goals make rehabilitation more expensive and more likely to fail. Similarly, ecological communities differ in the proportion of species that respond well in rehabilitation, making some communities much more challenging to return than others. Rehabilitation failure may result in negligible or significant consequences to operations, environments and society.

Where goal complexity and site attributes interact to create high risk of rehabilitation failure and, especially where failure has significant consequences, mine planners should pay very close attention to managing and improving practices that identify and then minimise risk. Where goal complexity arises from vegetation community or species-specific targets, this risk analysis requires significant input from ecological scientists. In some systems, ecological advice or research may enable identification of alternative targets that meet project goals with reduced complexity, e.g. by identifying a range of potential reference communities (Garrah \& Campbell 2011). Sometimes rehabilitation goals require an approach to landforming or site treatment that should involve planning at an earlier stage. Rehabilitation teams often do not have a voice in post-mining landforms and have limited capacity to alter surface materials or profiles after sites are handed over. This may be too late to make critical changes so there need to be greater role for ecological input throughout the closure process (De Jong et al. 2015).

Tools to quantify this ecology-associated risk are effectively absent, the quality of expert opinion is limited by availability of experienced analysts, and mining in many systems has few precedents of successful rehabilitation with complex goals from which valid extrapolation is possible. This kind of risk analysis should be undertaken at the project development phase and incorporated into considerations of project planning and feasibility. It is likely that, on analysis, some project rehabilitation goals may be determined to be very high risk or not achievable, and should not proceed. This knowledge should be available to investors, planners and regulators prior to commencement of works.

If closure goals include ecological attributes such as a specific ecological community, a specific vegetation structure or level of species diversity, weed cover or standard of resilience, it is critical that appropriate reference communities are identified and that they are properly assessed to derive realistic metrics. While this is simple to express, in reality the site selection, sampling design and measurement approaches employed to obtain reference data necessary to assess closure criteria are often inadequate. Poor reference site definition and sampling can even make derived closure standards harder to reach than required.

Similarly, approaches for sampling and monitoring rehabilitation should match those of the target sites if it is to be useful for assessing performance towards closure. Simultaneously developing protocols for surveyed reference sites and rehabilitation monitoring enhances the efficiency and usefulness of both. Deriving dynamic performance targets that reflect regeneration process following natural disturbance events such fire, can also provide insight on the likelihood or timeframes for rehabilitation to reach endpoint targets (Kirkman et al. 2013).

\section{Improving outcomes}

Investment in research to improve rehabilitation processes is perhaps the largest area of potential (Lamb et al. 2015). In a recent comprehensive analysis of science necessary to restore sustainable, resilient and biodiverse ecosystems, Kings Park scientists have identified 34 questions, covering areas from target setting and planning, sourcing biological materials, optimising plant establishment and growth and landscape integration and function. The goals of ecosystem restoration can differ from those of mining rehabilitation but most of the questions are relevant in different contexts; for instance: 1) To what extent might passive regeneration and dispersal achieve restoration targets? 2) Can species returns from topsoils be maximised 
through changing harvesting, storage respreading and treatment techniques? 3) Are restoration surface attributes impairing conditions for optimal growth and survival, can treatments mitigate these effects? 4) Can planting patterns or sequences be varied to optimise survival and growth in restoration? (Miller et al. submitted). These questions require the skills of ecologists, soil scientists, hydrologists, plant physiologists, seed scientists, horticultural scientists and conservation geneticists. This research can improve effectiveness and standards of rehabilitation outcomes, speed up timeframes, reduce costs and increase certainty and reduce risk associated with the likelihood of meeting completion criteria.

Lastly, ecological and conservation scientists should have a role in discussion about the appropriateness and conservation and ecological implication of different completion criteria and closure conditions. Questions such as what are the ecological consequences of restoring ecosystems with $70 \%$ of species richness versus $50 \%$ of species richness lie in the domain of ecology, or what is the relative cost of restoring this community versus that community. Advice on the difference in financial cost and risk of meeting these contrasting criteria may also require input from ecologists, together with mining and rehabilitation professionals (e.g. Erskine et al. 2007). Regulatory and approvals decisions that involve these questions are currently made largely on the basis of extrapolation and assumption. Improving the availability of data and the quality of assumptions in this area may enable more nuanced and more reasonable outcomes.

\section{References}

DeJong, JT, Tibbett, M \& Fourie, A 2015, 'Geotechnical systems that evolve with ecological processes', Environmental Earth Science, vol. 73, pp. 1067-1082.

Erskine, PD, Mulligan, DR, Olsen, MF, Kirsch, B \& Roe, P 2007, 'Completion criteria for rehabilitated forest ecosystems on mined land - should they be based on unmined reference sites?', in AB Fourie, M Tibbett \& JV Wiertz (eds), Proceedings of the Second International Seminar on Mine Closure, Australian Centre for Geomechanics, Perth, pp. 211-216.

Garrah, KL \& Campbell, D 2011, 'Reference conditions for rehabilitating mine stockpiles as novel upland ecosystems in Canada's subarctic', in AB Fourie, M Tibbett \& A Beersing (eds), Proceedings of the Sixth International Conference on Mine Closure, vol. 1: Mine Site Reclamation, Australian Centre for Geomechanics, Perth, pp. 11-17.

Kirkman, LK, Barnett, A, Williams, BW, Hiers, JK, Pokswinski, SM \& Mitchell, RJ 2013, 'A dynamic reference model: a framework for assessing biodiversity restoration goals in a fire-dependent ecosystem', Ecological Applications, vol. 23, no. 7, pp. $1574-1587$.

Lamb, D, Erskine, PD \& Fletcher, A 2015, 'Widening gap between expectations and practice in Australian minesite rehabilitation', Ecological Management \& Restoration, vol. 16, no. 3, pp. 186-195.

Tibbett, M (ed.) 2015, Mining in Ecologically Sensitive Landscapes, CRC Press, p. 276.

Williams, AV, Nevill, PG \& Krauss, SL 2014, 'Next generation restoration genetics: applications and opportunities', Trends in Plant Science, vol. 19, no. 8, pp. 529-537. 